\title{
EmERGE platform: a new mHealth solution for people living with HIV
}

\author{
Paloma Chausa ${ }^{10000-0002-6740-657 X]}$, Francisco J. Gárate ${ }^{10000-0002-3729-5282]}$, Cesar Cáce- \\ $\operatorname{res}^{1,3}[0000-0002-1546-775 \mathrm{X}]$, Edward Wallitt ${ }^{4}$, Jennifer Whetham ${ }^{5}$, Enrique J. Gómez ${ }^{1,2[0000-}$ \\ 0001-6998-1407] and the EmERGE Consortium \\ ${ }^{1}$ Biomedical Engineering and Telemedicine Centre, ETSI Telecomunicación, Center for \\ Biomedical Technology, Universidad Politécnica de Madrid, Madrid, Spain, \\ pchausa/fgarate/ccaceres/egomezegbt.tfo.upm.es \\ ${ }^{2}$ Centro de Investigación Biomédica en Red, Biomateriales y Nanomedicina (CIBER-BBN), \\ Madrid, Spain \\ ${ }^{3}$ Universidad Rey Juan Carlos, Madrid, Spain, cesar. caceres@urj c.es \\ ${ }^{4}$ Podmedics, United Kingdom, edwallitteme.com \\ ${ }^{5}$ Department of HIV/ Genito-Urinary Medicine, Brighton and Sussex University Hospitals \\ NHS Trust, United Kingdom, $j$. whethamenhs . net.
}

\begin{abstract}
The EmERGE platform is a novel mHealth solution that supports a new paradigm for HIV care. Following a rigorous co-design approach, it provides users with a system that can link securely into the health records of an individual, select the data required, and once reviewed by a clinician send the data and clinical opinion confidentially to the individual's smart device. The proposed clinical pathway aims to facilitate patient empowerment and self-management of HIV while reducing face-to-face consultations for people living with stable HIV. The EmERGE platform has been deployed at 5 European clinical sites and it is being successfully used by more than 2000 individuals. A formal evaluation is currently underway using a tailored Health Technology Assessment process specifically developed for the assessment of mHealth solutions. The evaluation will assess the impact of the EmERGE pathway on patient empowerment, quality of life, patient related outcomes and experience measures, usability, quality of care and cost in diverse health systems.
\end{abstract}

Keywords: mHealth, PLWH, Patient empowerment, Self-management, HIV, Health Technology Assessment, Co-design, Healthcare pathways.

\section{Introduction}

With the advent of effective antiretroviral therapy (ART) HIV is now regarded as a chronic illness with a normal life expectancy in individuals who have access to testing, treatment and care as in the European Union [1]. The majority of individuals have an undetectable HIV viral load (the aim of ART) on stable treatment and many are now seeking to reduce the impact of HIV on their lives. 
People living with HIV (PLWH) are currently seen by an HIV clinician every 3-6 months in accordance with European guidelines [2]. The number of new diagnoses has continued to increase in many parts over recent years; as people are living longer healthier lives the number of people needing clinical care continues to increase without a proportionate increase in resource. Therefore, it is imperative that new models of service provision are explored and evaluated.

Previous experiences have demonstrated that web-based telemedicine approaches can be considered as feasible and safe tools for the management of stable HIV infection [4]. Similarly, mHealth interventions have improved health outcomes and self-management when used by patients with chronic disease conditions [5]. Thus, mHealth supported pathways of care provide an opportunity to offer an alternative to traditional models care that can reduce appointments for individuals living with stable HIV and increase capacity to see those living with more complex disease.

The EmERGE project (Evaluating mHealth technology in HIV to improve Empowerment and healthcare utilisation: Research and innovation to Generate Evidence for personalised care, http://www.emergeproject.eu/) is a five year Horizon 2020 funded project which is assessing the impact of a co-designed mHealth supported pathway of care for people living with medically stable HIV in diverse settings across 5 European countries. Impact is being assessed in terms of patient empowerment, quality of life, person-centred symptoms and concerns, quality of care and costs. The EmERGE mHealth platform supporting the pathway integrates with clinic ICT systems to reduce face-to-face appointments in patients living with stable HIV and is described in more detail here.

\section{General description and EmERGE platform architecture}

Overall, the goal of the EmERGE platform is to provide relevant, timely and secure delivery of health care data to people living with stable HIV infection through the use of novel mHealth tools. The optimal features of the platform have been determined through co-design work with PLWH and clinicians [5,6].

The EmERGE platform consists of the Adaptor, the Mobile Application, the Clinical Web Application (CWA) and the Messaging Service. This group of software allows patient data to be gathered from the hospital network, formatted appropriately, and sent to and displayed on patient devices.

The basic purpose of the CWA is to provide clinical users with an interface to register and manage patient users and their mobile devices. In addition, the application provides executive control over delivery of information such as blood tests results, medication lists and appointment information to mobile devices. The function of the Mobile Application is to receive user specific information from the CWA. The Messaging Service is the bridge between the CWA and the patient Mobile Application. Medication information, results, and appointments for specific individuals are sent to the Messaging Service to be served to patient devices on request. The Adaptor provides 
a single API for the CWA to extract the data via an interface with each hospital database. It ensures that the data are in a single format for onward processing and facilitates future integration with other clinical systems.

The basic structure of the EmERGE platform and the interaction of the four key elements is shown in the schematic below (Figure 1).

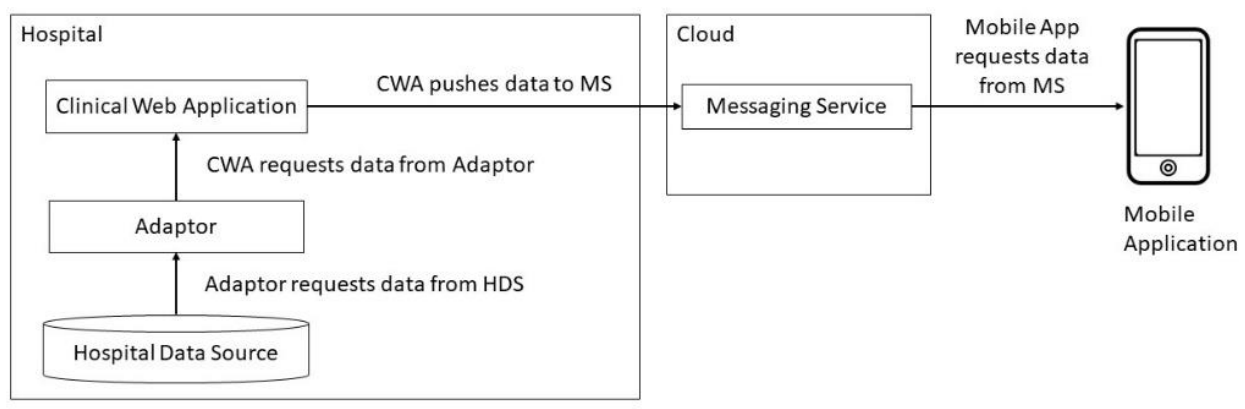

Fig. 1. EmERGE platform architecture

\section{EmERGE platform specification}

\subsection{Functional Requirements}

The main users of the EmERGE platform are the clinical and administrative staff of the clinical sites involved and PLWH - currently those enrolled in the clinical study designed to evaluate this new health care paradigm. Clinicians use the Clinical Web Application, a software application hosted inside the hospital network to provide the interface for the EmERGE system. The main functionalities of the CWA - as co-designed with clinicians - are described in the following table.

Table 1. Clinical Web Application functionalities.

\begin{tabular}{|c|c|}
\hline Functionality & Description \\
\hline Authentication & Unique username and password to login to the CWA \\
\hline Patient/device registration & Registration of patients and the associated mobile devices \\
\hline All patients visualization & Search and filtering of all registered patients \\
\hline Patient data visualization & $\begin{array}{l}\text { Overview of each patient user: current medication list (name, } \\
\text { dose/frequency and date of commencement), blood test re- } \\
\text { sults (graphically and in a table format) and appointment lists }\end{array}$ \\
\hline $\begin{array}{l}\text { 'Virtual clinics' appoint- } \\
\text { ment management }\end{array}$ & $\begin{array}{l}\text { Send data to the mobile application; re-book the patient for a } \\
\text { future revision; pause the patient in the system }\end{array}$ \\
\hline Messaging to patients & Send messages to a specific patient \\
\hline Edition of FAQs & Provision of Frequently Ask Questions \\
\hline Users management & Creation and elimination of users and assignment of roles \\
\hline
\end{tabular}


PLWH use the Mobile Application to receive their data including laboratory results, information about the treatment and appointments with the aim of empowering individuals, to self-manage their infection. The main functionalities of the Mobile Application - as co-designed with the community - are described in the following table.

Table 2. Mobile Application functionalities.

\begin{tabular}{|c|c|}
\hline Functionality & Description \\
\hline Authentication & $\begin{array}{l}\text { Patient Users are required to login to the application on } \\
\text { each use through passcode or biometrics. }\end{array}$ \\
\hline Medication visualization & $\begin{array}{l}\text { Visualization of medication list (name of medication, } \\
\text { dose/frequency and date of commencement). Link to Liv- } \\
\text { erpool HIV drugs interaction webpage }\end{array}$ \\
\hline $\begin{array}{l}\text { Laboratory Results } \\
\text { visualization }\end{array}$ & $\begin{array}{l}\text { Visualization of blood test results: current and historical } \\
\text { data; evolution graphs }\end{array}$ \\
\hline Appointments & $\begin{array}{l}\text { Visualization of next appointments. Integration with pa- } \\
\text { tient mobile calendar }\end{array}$ \\
\hline Messages & Messaging from Hospital clinicians \\
\hline Help desk and information & Support and access to FAQs \\
\hline Application Settings & $\begin{array}{l}\text { Erase application data, change passcode, change security } \\
\text { question, ask for a new password, study withdrawal }\end{array}$ \\
\hline
\end{tabular}

\subsection{Technology used}

The Mobile Application has been built using a hybrid approach provided by the Ionic Framework (http://ionicframework.com). A hybrid approach enables creation of native iPhone and Android applications from a single and maintainable codebase. Apps can be built with Web technologies provided by Ionic (CSS, HTML5, Sass, etc.) and then distributed through native app stores to be installed on devices. For Android, Ionic supports Android 4.1 and up. For iOS, Ionic supports iOS 7 and up.

Both the Clinical Web Application and the Adaptor are implemented using Ruby on Rails version 4.2 and Ruby version 2.3. Ruby on Rails (https://rubyonrails.org/), is an open-source, mature and well-maintained server-side web application framework written in Ruby. Ruby on Rails is a model-view-controller (MVC) framework, providing default structures for a database, a web service, and web pages. It encour-ages and facilitates the use of web standards such as JSON or XML for data transfer, and HTML, CSS and JavaScript for display and user interfacing.

Heroku (https://www.heroku.com/) is used to host the Messaging Service. Heroku is a cloud platform as a service (PaaS) supporting several programming languages. It lets the developer build, run and scale applications. Apart from the production MS, there is a full staging version system set up on Heroku for showing off the system and testing them. 


\subsection{User Interfaces and graphical design}

The EmERGE platform offers two main User Interfaces: the Clinical Web Application for clinicians and the Mobile Application for PLWH. The CWA has been built using bootstrap-sass 3 for the graphical design. Bootstrap is a popular open-source framework that allows web designers of all skill levels to quickly build a site. This version of Bootstrap uses Sass (https://sass-lang.com) as the pre-processor of choice. Sass (Syntactically Awesome Style Sheets) is a pre-processor scripting language that is interpreted or compiled into Cascading Style Sheets (CSS). The Mobile Application also uses Sass for the graphical design. Figure 2 shows the CWA and Mobile Application interfaces.

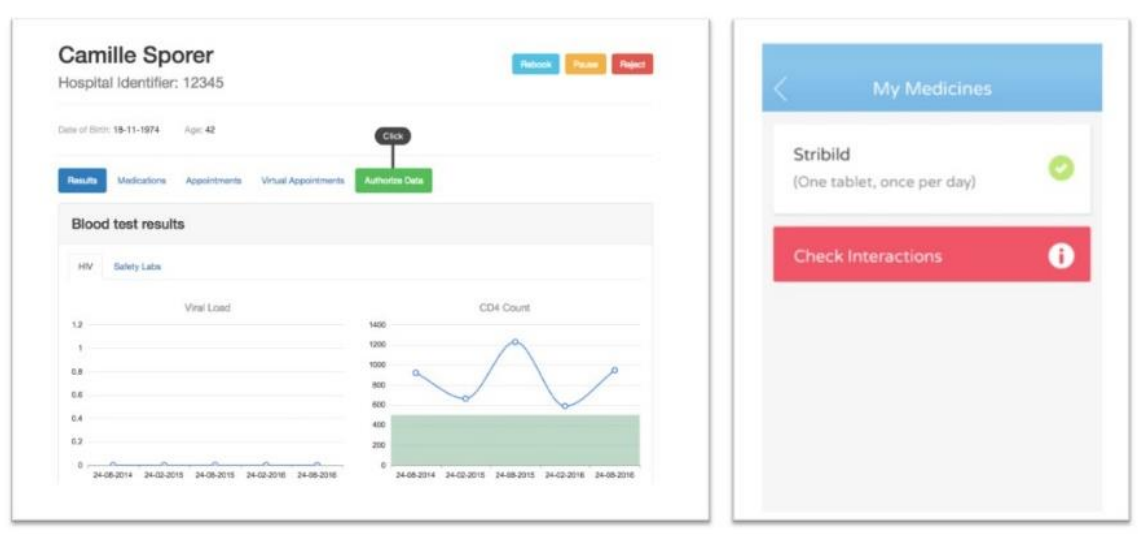

Fig. 2. - CWA and Mobile Application User Interfaces

\subsection{Security and Privacy}

Security is a key factor for our patient group and for their clinicians. The CWA within each site is to be regarded as a 'walled-off data silo'. It exists within the existing IT infrastructure of the clinical site and should not be open for external access. As such, unless connected via a VPN, access to the CWA is not possible outside of the hospital network.

All clinical and demographic data stored locally within the CWA's local database are encrypted to a minimum of 256bit AES. Transmission of data outside of the hospital network, from the CWA to the Mobile Application is encrypted over Secure Sockets Layer (SSL) and includes no patient identifying information.

\section{EmERGE platform evaluation procedure}

A large prospective cohort study, undertaken in five European sites is being performed to validate the EmERGE platform supported pathway of care using a tailored Health Technology Assessment (HTA) process, Model for Assessment of Telemedicine Applications (MAST) and will assess impact in terms of patient empowerment, quality of 
life, patient related outcome and experience measures; health economics, sociotechnical aspects and clinical outcomes.

The five clinical sites involved in the study are HIV clinics in Antwerp, Barcelona, Brighton, Lisbon and Zagreb. The general care of PLWH is very similar in the five clinical sites, specifically in the follow up of individuals living with stable HIV. PLWH who are participating in the study have visits at baseline, month 6, 12, 18, 24 and 30. At baseline, participants visit the hospital for a face-to-face visit and are introduced to the EmERGE mobile application. At six months the individual will attend for bloods tests as per standard of care. The results will be checked by a clinician and then be pushed through to the patient's mobile phone if the results are unremarkable. Visits at 18 and 30 months will be conducted in a similar fashion. At 12 months and 24 months the patient will be seen by their clinical team in person (or virtually if normally cared for within the Barcelona Virtual Hospital).

The recruitment of patients started on April 2017 and the platform is successfully being used by 2251 patients and more than 20 clinicians. Specifically, the evaluation includes the assessment of the impact of the EmERGE platform on the items listed in the Table 3 .

Table 3. Evaluation of EmERGE platform.

\begin{tabular}{ll}
\hline Variable & Evaluation Procedure \\
\hline Self-management and & Patient Activation Measure (PAM-13) questionnaire at baseline, \\
empowerment & months 12 and 24. \\
Acceptability/ usability & Satisfaction System Usability Scale (SUS) at months 12 and 24 \\
& CD4 count, viral load, percentage of patients with changes in \\
Clinical Outcomes & ART) at months 6, 12, 18, 24 and 30 \\
Quality of Life & EQ-5D-5L, PROQOL-HIV questionnaires at baseline, months \\
& 12 and 24 \\
Adherence & Modified Medication Adherence Self-Report Inventory (M- \\
& MASRI) questionnaire at baseline, months 12 and 24 \\
\hline
\end{tabular}

An electronic Case Report Form (eCRF) has been developed to collect patient questionnaires and the clinical related data. It has been built using Ruby on Rails and PostgreSQL as Data Management System. The eCRF is hosted by Heroku and all the information is encrypted by means of bcrypt.

\section{Conclusions}

The EmERGE platform supports a novel pathway of care for individuals living with stable HIV infection. In this paper we describe the technology that has been developed and integrated, and is currently being used by individuals, in diverse care settings across five European countries.

This technology has been co-designed with community and clinicians [5,6] and a formal evaluation with over 2000 individuals is currently underway using a tailored 
Health Technology Assessment process, specifically developed for the assessment of mHealth solutions.

The final goal aims to demonstrate the benefits to patients and simultaneous increases in cost-effectiveness for healthcare providers by reducing face-to-face consultations: assessing the intervention in terms of patient empowerment, quality of life, patient related outcomes and experience measures, usability, quality of care and cost.

Acknowledgements. This project has received funding from the European Union's Horizon 2020 research and innovation programme under grant agreement No 643736. The dissemination activities within the EmERGE project do not represent the opinion of the European Community and only reflects the opinion of the authors. The scientific members of the EmERGE consortium are: Ludwig Apers; Eduard Beck; Josip Begovac; Margarida Borges; Stephen Bremner; Paloma Chausa; Mary Darking; Maria Dutarte; Duncan Fatz; Steven Hoornaert; David García; Felipe García; Enrique J. Gomez; Flis Henwood; Agathe Leon; Sundhiya Mandalia; Benjamin Marent; Rosa Muñoz; Gonçalo Rodrigues; Eugenio Teofilo; Brian West; Jennifer Whetham; Platonas Yfantopoulos; Sime Zekan. Our thanks also to our advisory board which includes: Cesar Caceres; Richard Harding \& Edward Wallitt.

\section{References}

1. May M et al; AIDS. 2014 Feb 19. [Epub ahead of print]; Impact on life expectancy of HIV1 positive individuals of $\mathrm{CD} 4+$ cell count and viral load response to antiretroviral therapy: UK cohort study.

2. EACS guidelines version 9.1 October 2018 http://www.eacsociety.org/files/2018_guidelines-9.1-english.pdf last accessed 25/04/2019

3. Agathe León, César Cáceres, Emma Fernández, Paloma Chausa, Maite Martin, Carles Codina, Araceli Rousaud, Jordi Blanch, Josep Mallolas, Esteban Martinez, Jose L. Blanco, Montserrat Laguno, Maria Larrousse, Ana Milinkovic, Laura Zamora, Neus Canal, Josep M. Miró, Josep M. Gatell, Enrique J. Gómez, Felipe García. A New Multidisciplinary Home Care Telemedicine System to Monitor Stable Chronic Human Immunodeficiency Virus-Infected Patients: A Randomized Study. PLoS ONE, 1932-6203, January, 2011

4. Lee JA, Choi M, Lee SA, Jiang N. Effective behavioral intervention strategies using mobile health applications for chronic disease management: a systematic review. BMC Med Inform Decis Mak. 2018;18(1):12. Published 2018 Feb 20. doi:10.1186/s12911-018-0591-0

5. Marent, B., Henwood, F., Darking, M., on behalf of the EmERGE Consortium (2018) 'Ambivalence in digital health: Co-designing an mHealth platform for HIV care'. Social Science \& Medicine 215, pp 133-241.

6. Marent, B., Henwood, F., Darking, M., EmERGE Consortium (2018) 'Development of an mHealth platform for HIV care: Gathering user perspectives through co-design workshops and interviews'. Journal of Medical Internet Research http://doi.org/10.2196/preprints.9856 